\title{
Video Article \\ Prescribed 3-D Direct Writing of Suspended Micron/Sub-micron Scale Fiber Structures via a Robotic Dispensing System
}

\author{
Hanwen Yuan ${ }^{1}$, Scott D. Cambron ${ }^{1}$, Robert S. Keynton ${ }^{1}$ \\ ${ }^{1}$ Bioengineering Department, University of Louisville \\ Correspondence to: Robert S. Keynton at robert.keynton@louisville.edu \\ URL: https://www.jove.com/video/52834 \\ DOI: doi: $10.3791 / 52834$
}

Keywords: Engineering, Issue 100, Direct write, precise control, micro/sub-micron scale fibers, 3-axis robot, dispensing system

Date Published: 6/12/2015

Citation: Yuan, H., Cambron, S.D., Keynton, R.S. Prescribed 3-D Direct Writing of Suspended Micron/Sub-micron Scale Fiber Structures via a Robotic Dispensing System. J. Vis. Exp. (100), e52834, doi:10.3791/52834 (2015).

\section{Abstract}

A 3-axis dispensing system is utilized to control the initiating and terminating fiber positions and trajectory via the dispensing software. The polymer fiber length and orientation is defined by the spatial positioning of the dispensing system 3-axis stages. The fiber diameter is defined by the prescribed dispense time of the dispensing system valve, the feed rate (the speed at which the stage traverses from an initiating to a terminating position), the gauge diameter of the dispensing tip, the viscosity and surface tension of the polymer solution, and the programmed drawing length. The stage feed rate affects the polymer solution's evaporation rate and capillary breakup of the filaments. The dispensing system consists of a pneumatic valve controller, a droplet-dispensing valve and a dispensing tip. Characterization of the direct write process to determine the optimum combination of factors leads to repeatedly acquiring the desired range of fiber diameters. The advantage of this robotic dispensing system is the ease of obtaining a precise range of micron/sub-micron fibers onto a desired, programmed location via automated process control. Here, the discussed self-assembled micron/sub-micron scale 3D structures have been employed to fabricate suspended structures to create micron/sub-micron fluidic devices and bioengineered scaffolds.

\section{Video Link}

The video component of this article can be found at https://www.jove.com/video/52834/

\section{Introduction}

Over the past several decades, a variety of fabrication techniques, such as wet spinning, dry spinning and electrospinning, have been employed to create novel polymer fiber structures with diverse and robust biological, chemical, electrical and mechanical properties ${ }^{1-12}$. Although these spinning techniques are capable of generating suspended three-dimensional fibers, they are limited in their ability to precisely control fiber orientation in three dimensions since fiber deposition via these processes are random in nature. In addition, these techniques are restricted in their dimensional range for fiber fabrication; specifically, fibers produced via wet and dry spinning vary in diameter from tens to hundreds of microns, while electrospinning yields fibers with diameters ranging from tens of nanometers to a single micron ${ }^{13}$.

To provide more precise control of fiber orientation in 3-D space, our group developed a self-assemble or "direct-write" fiber fabrication process that directly ejects a polymeric material out of a hollow capillary and then draws individual filaments which thin and solidify into predictable fiber diameters by exploiting surface tension-driven fluid mechanics ${ }^{14}$. Our initial direct-write system for increasing the level of control of fiber position and diameter consisted of a custom fabricated spring loaded syringe dispensing system attached to the head of a custom made Ultra-High Precision Micromilling Machine (Figure 1). The UHPMM had a stage with a positional resolution of $1.25 \mathrm{~nm}$ in the $X$ and $Y$ directions and 20 $\mathrm{nm}$ in the $Z$ direction that was programmatically controlled to create micron and sub-micron scale wires and structures. One limitation of this particular direct write system was the lack of flow control of the polymer solution through the needle tip. Although the spring loaded dispensing system successfully generated constant flow through the tip, a continuously expanding spherical bead of polymer solution was created at the outlet of the syringe tip, which varied in size and volume depending on environmental conditions. 


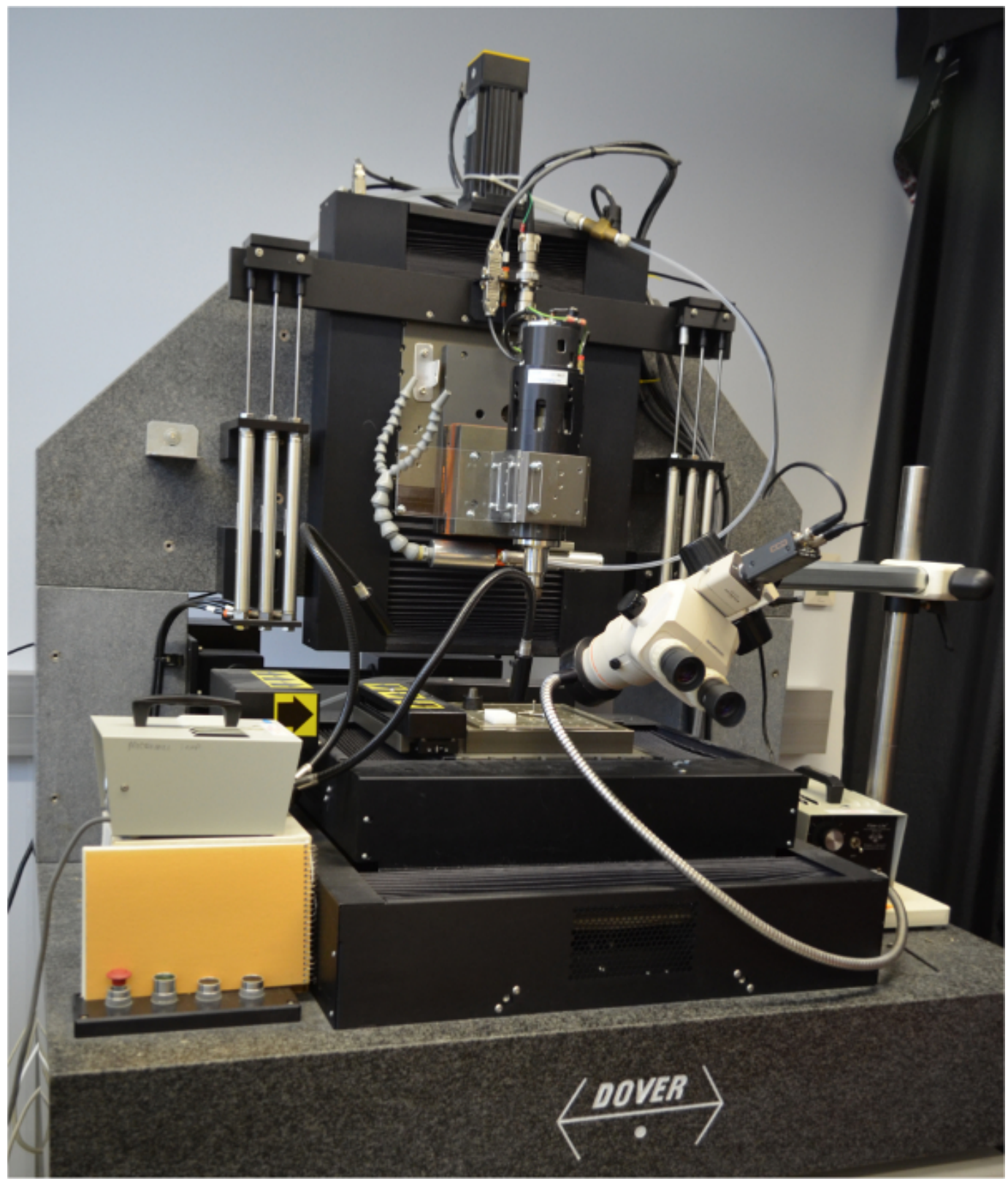

Figure 1. Image of Ultra High Precision Micromilling Machine: the first direct write system employed in fabricating micron/sub-micron scale structures. Please click here to view a larger version of this figure.

The inconsistency of this source bead impacted the ability of the system to repeatedly fabricate wires of a prescribed diameter. Albeit, structures were successfully generated using this direct write process, enhancement of the process by increasing control of the flow of polymer solution would allow for more precise, prescribed fiber diameters through regulation of the bead size at the syringe tip. Thus, this work describes the implementation of a 3-axis automated dispensing system with pneumatically actuated dispenser valve to precisely control the polymer solution flow rate and tip bead size to create prescribed, micron/sub-micron suspended structures.

\section{Equipment Set-up}

1. Assemble dispensing system and connect valve controller and syringe barrel to the pneumatic source, via a pressure regulator, to set the pressure at $15 \mathrm{psi}$ to dispense the polymer solution from the needle tip at a flow rate of $2.45 \mu \mathrm{l} / \mathrm{min}$.

2. Insert the 3-axis robot and dispensing system into a thermal enclosure to ensure a stable working environment (Figure 2).

3. Install Joint-Robot Control Points (JR-C Points) software provided by the system manufacturer and connect 3-axis robot to a computer via serial communication port.

4. Mount dispensing valve to 3 -axis robot and install needle tip onto the valve.

5. Level the robotic stage platen according to the manufacturer's guidelines to ensure flatness with respect to the height of the valve tip ${ }^{15}$. 
6. Assemble a feedback-controlled heater in the enclosure to add environmental control.

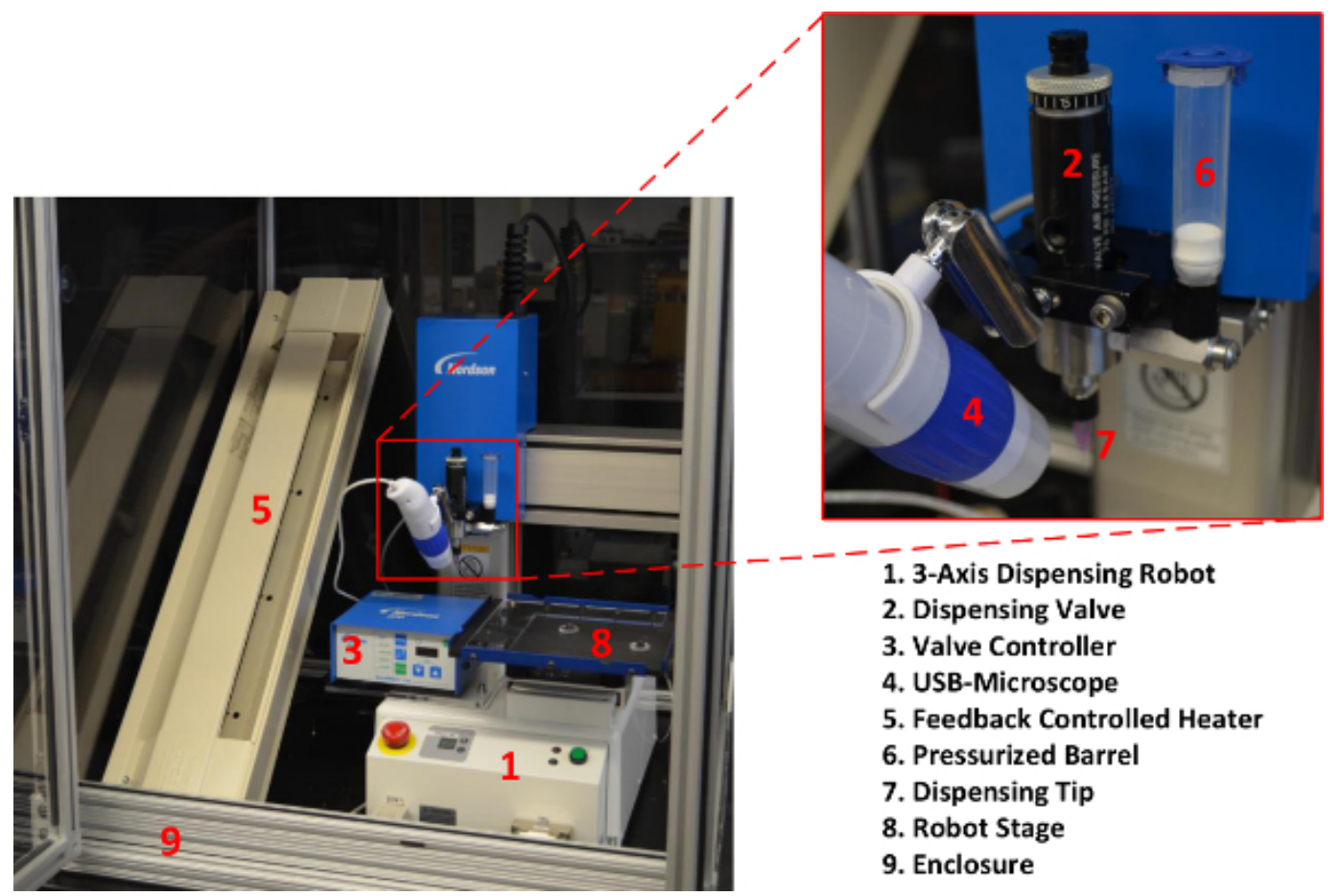

Figure 2. (A) 3-Axis dispensing robot with required accessories housed inside an enclosure; and, (B) close up image of the dispensing valve with attached USB microscope for visualization. Please click here to view a larger version of this figure.

\section{Experimental Materials and Controlling Factors}

1. Controlling factors and their combination.

1. Vary feed rate from $1 \%$ speed $(5 \mathrm{~mm} / \mathrm{sec})$ up to $100 \%(500 \mathrm{~mm} / \mathrm{sec})$, by the JR C-Points software. For this application, use a $2 \%$ speed $(10 \mathrm{~mm} / \mathrm{sec})$ to fabricate the fiber structures presented in this work.

2. Vary polymer solution concentrations to attain specific viscosity, surface tension and volatility parameters required for desired application. For this application, use a $24 \%$ polymethylmethacrylate (PMMA) in Chlorobenzene solution to fabricate the fiber structures presented in this work.

3. Vary control valve dispense time from $0.02 \mathrm{sec}$ to $1 \mathrm{sec}$ in order to eject solutions via pneumatic air. For this application, use $0.02 \mathrm{sec}$ to fabricate the fiber structures presented in this work.

4. Select a gauge size of high precision needle tips to dispense polymer solutions. For this application, use a $30 \mathrm{G}$ (inner diameter (ID) = $152.4 \mu \mathrm{m}$ ) tip to fabricate the fiber structures presented in this work.

5. Set the operating temperature range between $70^{\circ} \mathrm{F}$ to $100^{\circ} \mathrm{F}$ in the thermally insulated box to maintain a constant evaporation rate of the polymer sample. For this application, use a temperature of $70^{\circ} \mathrm{F}$ to fabricate the fiber structures presented in this work.

2. Preparation of polymer solutions.

1. Mix polymer resins of polymethyl methacrylate (PMMA; $0.72 \mathrm{~g})$ with the solvent Chlorobenzene $(2.28 \mathrm{~g})$ under a chemical flow hood.

2. Calculate the weight of the polymer (PMMA) and its solvent (chlorobenzene) to achieve the desired concentration of polymer in solution. For this application, use a $24 \%$ polymethylmethacrylate (PMMA) in Chlorobenzene solution to fabricate the fiber structures presented in this work.

3. Place desired amount of polymer powder/resin into glass vial. For this application, use $0.72 \mathrm{~g}$ of PMMA resin to attain a $24 \%$ concentration of PMMA.

4. Transfer solvent onto polymer within vial by pipette until the total weight of $3 \mathrm{~g}$ is reached.

5. Mix the vial for $1 \mathrm{~min}$ using a vortex shaker and ultrasonically process them for $5 \mathrm{hr}$ to completely dissolve the polymer powder/resin.

6. Check the transparency of solutions, if there is any cloudiness or opaqueness to sample continue to sonicate until clear.

3. Viscosity measurement of polymer solutions.

1. Measure solution viscosities via cone-and-plate viscometer (e.g., LVDV-II+ and RVDV-II++) ${ }^{16}$.

2. Load $0.5 \mathrm{ml}$ of known viscosity standard sample (a glycerin-based and water mixture) provided by the viscometer manufacturer to calibrate the viscometer in order to minimize errors in the viscosity measurements. The manufacturer provided a variety of standard samples for calibrating the viscometer. Use the glycerin-based standard sample with a viscosity of 100,000 cP.

3. Connect water jacket to viscometer to maintain the test fluid at a constant operating temperature.

4. Start test fluid measurement by moving the On/Off switch to the On position to initiate rotation of the spindle. Once the value of the torque on the display panel has stabilized, record the torque and calculate the final viscosities using the relationship between torque, spindle multiplier constant and speed of spindle ${ }^{16}$ 
$\eta_{\mathrm{s}}=T K \times S M C \times \frac{10000}{R P M} \quad(1)$

where is viscosity, RPM is the speed of the spindle affixed to cone, TK is the torque (0.09373 for LVDV-II+, 1 for RVDV-II+), SMC is the spindle multiplier constant that depends on the specific spindle being used during the viscosity measurement. For this study, the CP-52 spindle was used which has a spindle multiplier constant of 9.83 .

4. Surface tension measurement of polymer solutions.

1. Measurement method refers to the Wilhelmy technique. ${ }^{14}$

2. Place $1 \mathrm{ml}$ test solutions in a glass vial onto a high-resolution balance (scale resolution $=0.001 \mathrm{~g}$ ). Dip a glass rod of known diameter into test solutions.

3. Control the position of rod via a servo or stepper controlled linear actuator to touch surface and partially immerse into test solutions with zero contact.

4. Monitor and record the change in mass measurement on the balance when removing rod from solution surface.

5. Calculate surface tension by force change, perimeter of rod and contact angle of fluid on the rod.

$$
\sigma=\frac{F}{l \cos \theta}(2)
$$

where is the wetted perimeter of rod $(I=10.05 \mathrm{~mm}$ with diameter $=3.2 \mathrm{~mm}), \theta$ is contact angle of fluid on the rod, $F$ is force change due to surface tension of test fluid on the tip of rod.

5. Mass transfer coefficient measurement of solutions.

1. Measure mass transfer coefficients of polymer solutions by thermogravimetric analysis. ${ }^{14}$

2. Load $30 \mu \mathrm{l}$ of the $24 \%$ (by weight) PMMA polymer solution onto a platinum plate before taring the balance.

3. Vent the chamber, program the device to run at desired operating temperature $\left(70^{\circ} \mathrm{F}\right)$ for $2 \mathrm{hr}$ to monitor mass of target solutions.

4. Calculate the mass transfer coefficients by changes in the solution's mass, area of solution/air interface and density.

$$
\chi=\frac{-m(t) m(t)}{A\left(m(t)-m_{P O L Y M E R}\right) \rho}
$$

where $\mathrm{m}(\mathrm{t})$ is the mass of PMMA solutions, $\mathrm{A}$ is the area of the solution/air interface (equals $78.5 \mathrm{~mm}^{2}$ for standard plate), $m P O L Y M E R$ is the polymer mass in the solution and is the density of the solution.

\section{Direct Write Experiment Procedure}

1. Dispensing system sample loading protocol.

1. Load $3 \mathrm{ml}$ of polymer solution into the syringe barrel, place piston into syringe barrel to eliminate inconsistent pneumatic pressure distribution.

2. Twist the inlet line adapter on the syringe barrel, connect it to the source line air tube.

3. Choose desired gauge size of the precision needle tip to perform experiment.

4. On the dispense controller panel, switch to purge state and click "cycle" button to fill the dispense valve with polymer solution until discharged from the needle tip.

5. Wipe residual polymer solution from the tip in preparation of a programmed fiber writing procedure.

2. Direct write polymer fibers with 3 -axis robot and dispensing system.

1. Determine the robot stage offset to reposition the tip of the dispensing needle from the default home position to the initiation point of the prescribed micro/sub-micron fiber structure to be drawn. Mount a USB microscope (magnification $=200 \mathrm{X}$ ) to the dispensing system valve bracket, which translates along the Z-axis. Manually focus on the dispenser tip by adjusting the focusing knob on the USB microscope that assists in precisely positioning the valve tip to the desired location, prefabricated substrate or device.

2. Create/design the desired fiber structure pattern (Figures $\mathbf{3 A}, \mathbf{4 A}$, and $\mathbf{5 A}$ ) using a CAD software package. Input the spatial coordinates $(x, y, z)$ in the robot JR-C controlling software for all initiation and termination points in sequential order with respect to the desired pattern created within the CAD program (Figure 3B, 4B, and $5 B$ ). ${ }^{15}$

3. Transfer the completed fiber structure design program from the computer to the robot by clicking "Send C\&T Data" under the Robot menu within the JR-C software ${ }^{15}$.

4. Load $3 \mathrm{ml}$ of PMMA solution sample of known concentration (24\%) into syringe barrel, purge valve and needle, set all the dispensing parameters for the valve controller and robot controlling software.

5. Place prefabricated substrate onto the robot stage platen, close the thermal enclosure doors to prevent ambient air flow, which can induce unstable evaporation of the polymer solution.

6. Start writing the fibers onto the substrate by clicking the robot menu from JR-C software and selecting "test running" 15

7. DC sputter coat a conductive gold metal layer for 2 min until a $2 \mathrm{~nm}$ thick layer of gold is deposited onto the drawn fibers to allow visualization of the fibers in the scanning electron microscope.

8. Measure the diameter and structure of fibers via scanning electron microscope ${ }^{17}$. Microscope parameters: High voltage level: $2.00 \mathrm{kV}$; Objective: InLens, Working Distance $(9.0 \mathrm{~mm})$.

3. Perform the dispensing system cleaning procedure under a chemical flow hood

1. Place the dispensing system in a chemical flow hood to perform the cleaning procedure.

2. Disassemble needle valve according to manufacturer's protocol.

3. Place all the metal parts into a beaker, pour acetone into the beaker until all parts are immersed.

4. Place beaker in an ultrasonic bath for $30 \mathrm{~min}$ to remove all polymer remnants. 
5. Rinse all parts under flowing DI water, then use air gun to blow them dry.
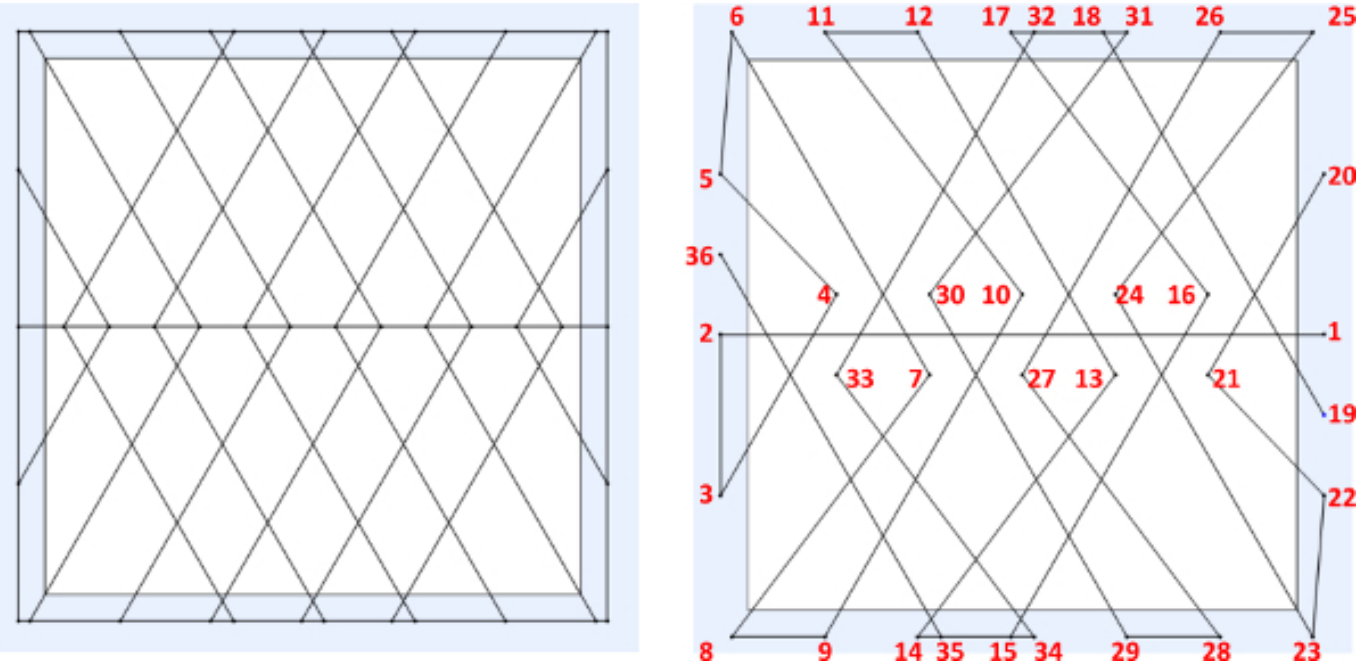

Figure 3. (A) Example of designed "quad" web structure on a $10 \mathrm{~mm} \times 10 \mathrm{~mm}$ frame, (B) sequential point-to-point spatial instructions to be inputted into JR-C point software. Please click here to view a larger version of this figure.
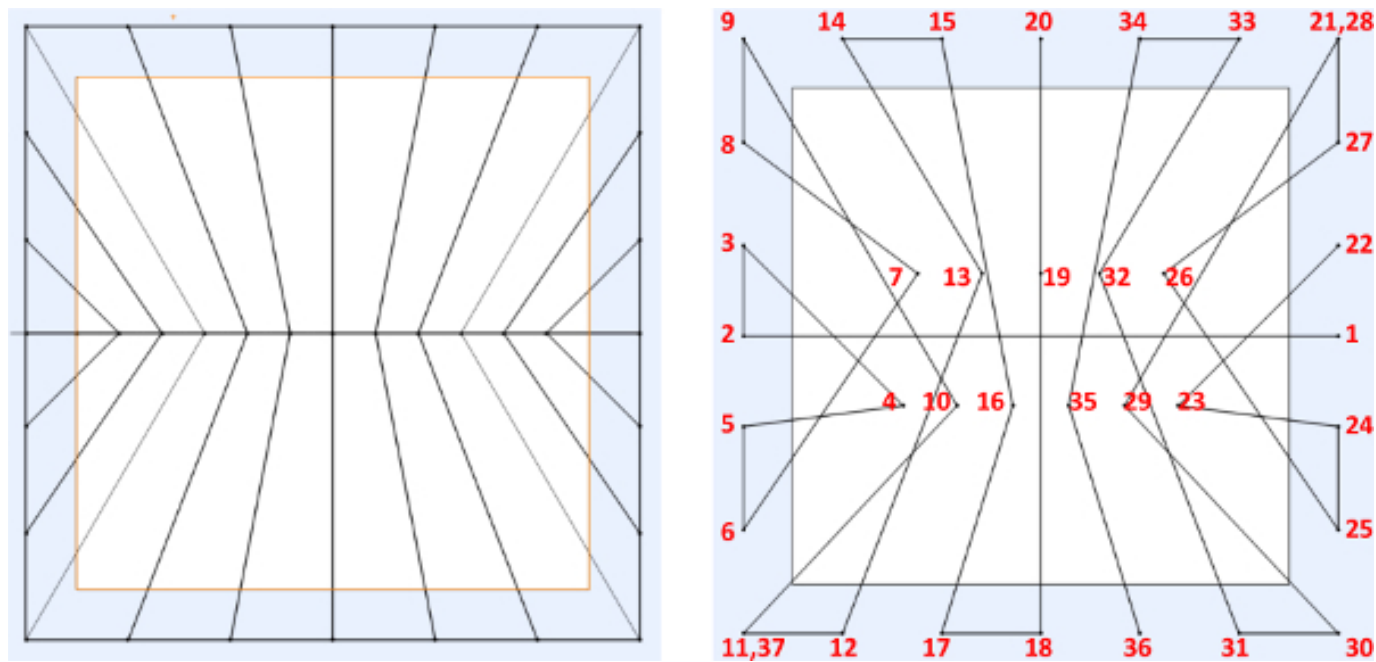

Figure 4. (A) Example of designed "symmetric" web structure on a $10 \mathrm{~mm} \times 10 \mathrm{~mm}$ frame, (B) sequential point-to-point spatial instructions to be inputted into JR-C point software. Please click here to view a larger version of this figure. 

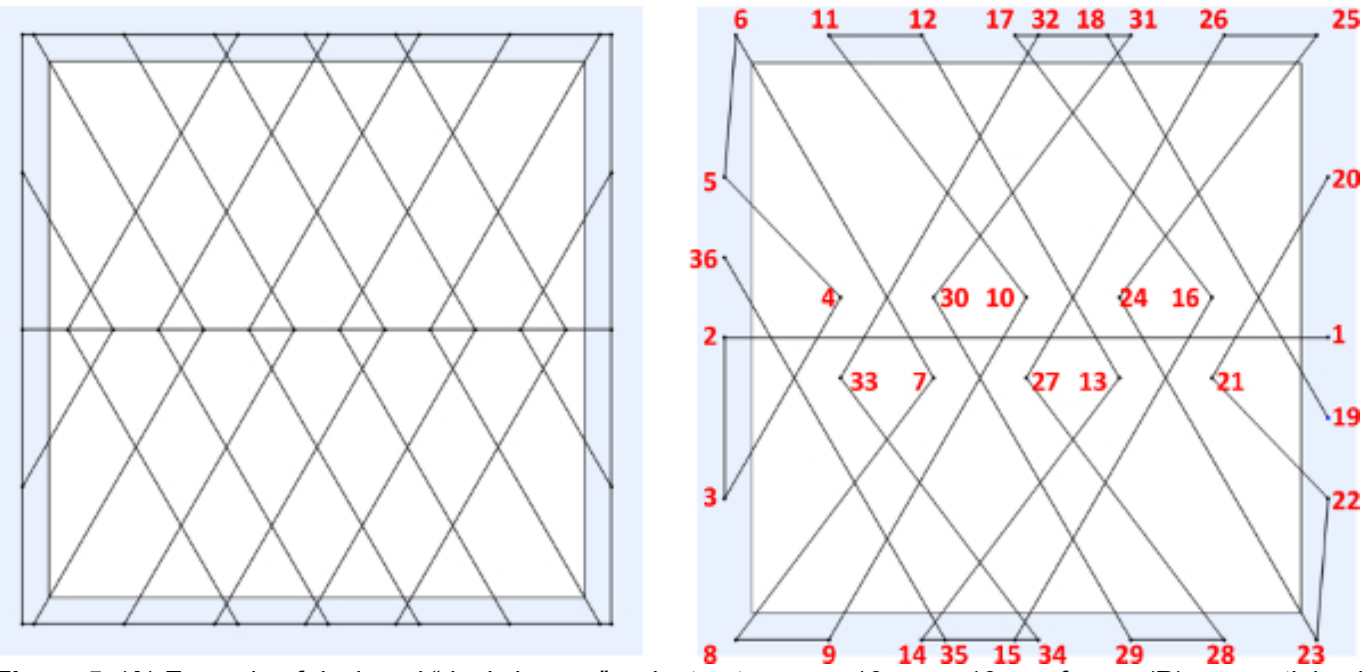

Figure 5. (A) Example of designed "dual chevron" web structure on a $10 \mathrm{~mm} \times 10 \mathrm{~mm}$ frame, (B) sequential point-to-point spatial instructions to be inputted into JR-C point software. Please click here to view a larger version of this figure.

\section{Representative Results}

Images of the actual structures produced via the 3-axis robot and direct write method for each of the respective designs and JR C-Point algorithms defined in Figures 3-5A \& B above are shown in Figures 6A, 7A \& 8A. As can be seen in the resulting images, three-dimensional, freely suspended fibers have been successfully "written" onto the substrates by precisely manipulating the dispenser tip to prescribed spatial locations including the initiating/terminating points as well as points of intersection. The insets of these figures magnify the intersection points of suspended fibers, which demonstrate the system's ability to precisely control fiber orientation (Figures 6B, 7B \& 8B) in 3-D space.

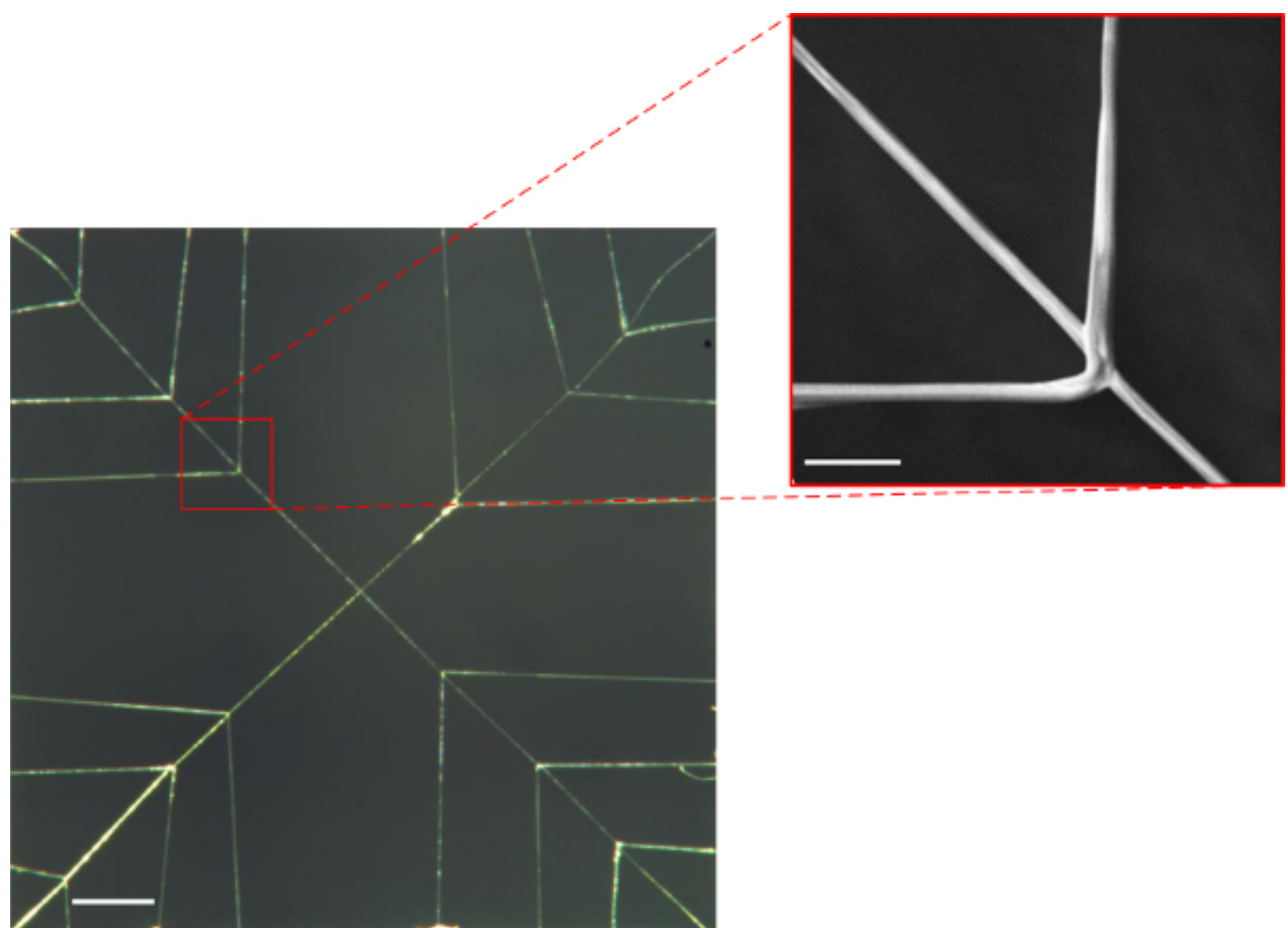

Figure 6. (A) Optical image of fabricated "quad" suspended web structure having 2 support fibers (diagonals) and 12 bifurcated branched PMMA fibers (15X optical magnification; scale bar $=1 \mathrm{~mm}$ ), (B) SEM image of a single bifurcated fiber (289X magnification; scale bar = $100 \mu \mathrm{m})$. Please click here to view a larger version of this figure. 


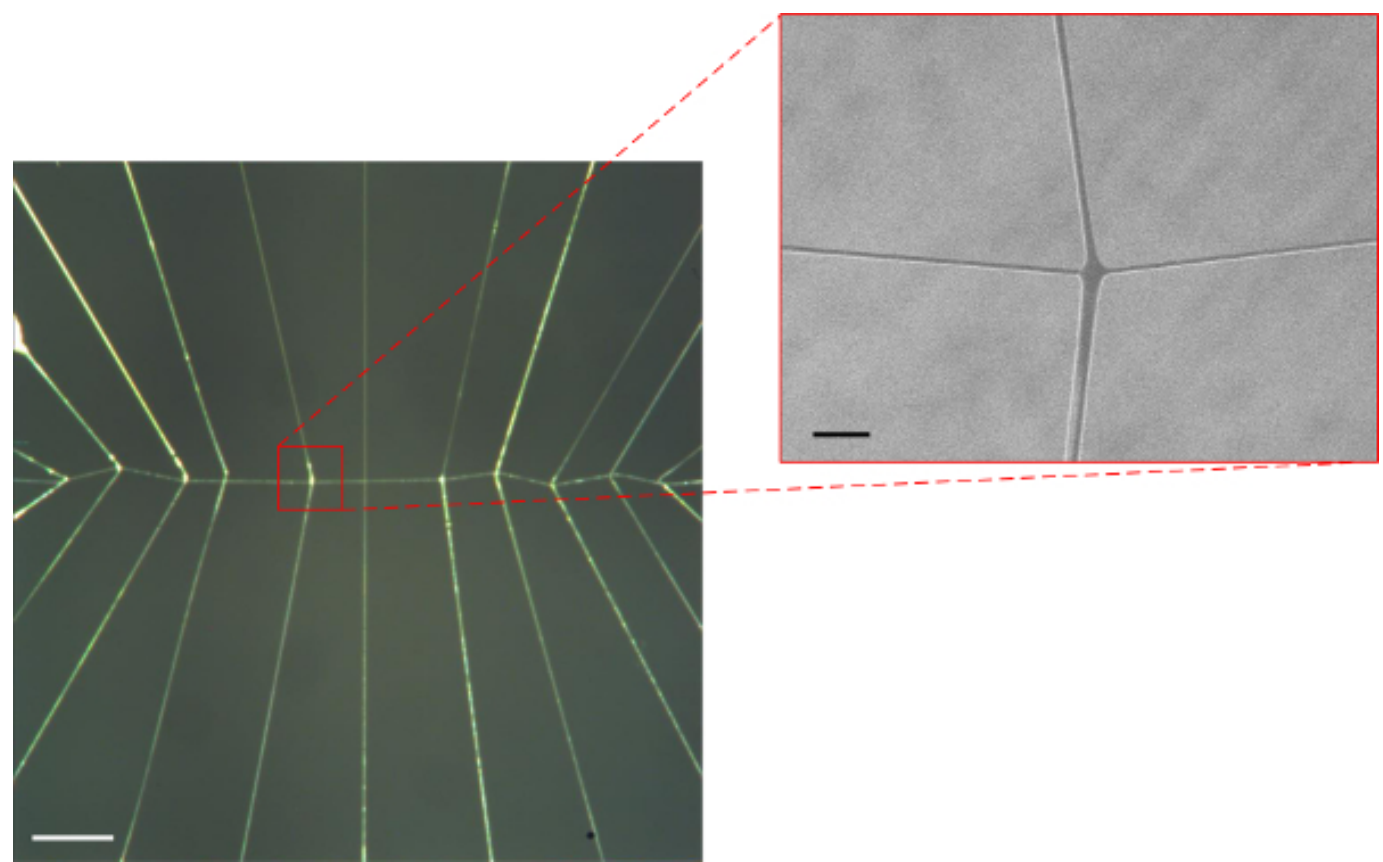

Figure 7. (A) Optical image of fabricated "symmetric" suspended web structure having 1 support fiber (horizontal) and 11 bifurcated branched PMMA fibers (15X optical magnification; scale bar $=1 \mathrm{~mm}$ ), (B) SEM image of a single bifurcated fiber $(107 \mathrm{X}$ magnification; scale bar $=100 \mu \mathrm{m})$. Please click here to view a larger version of this figure.

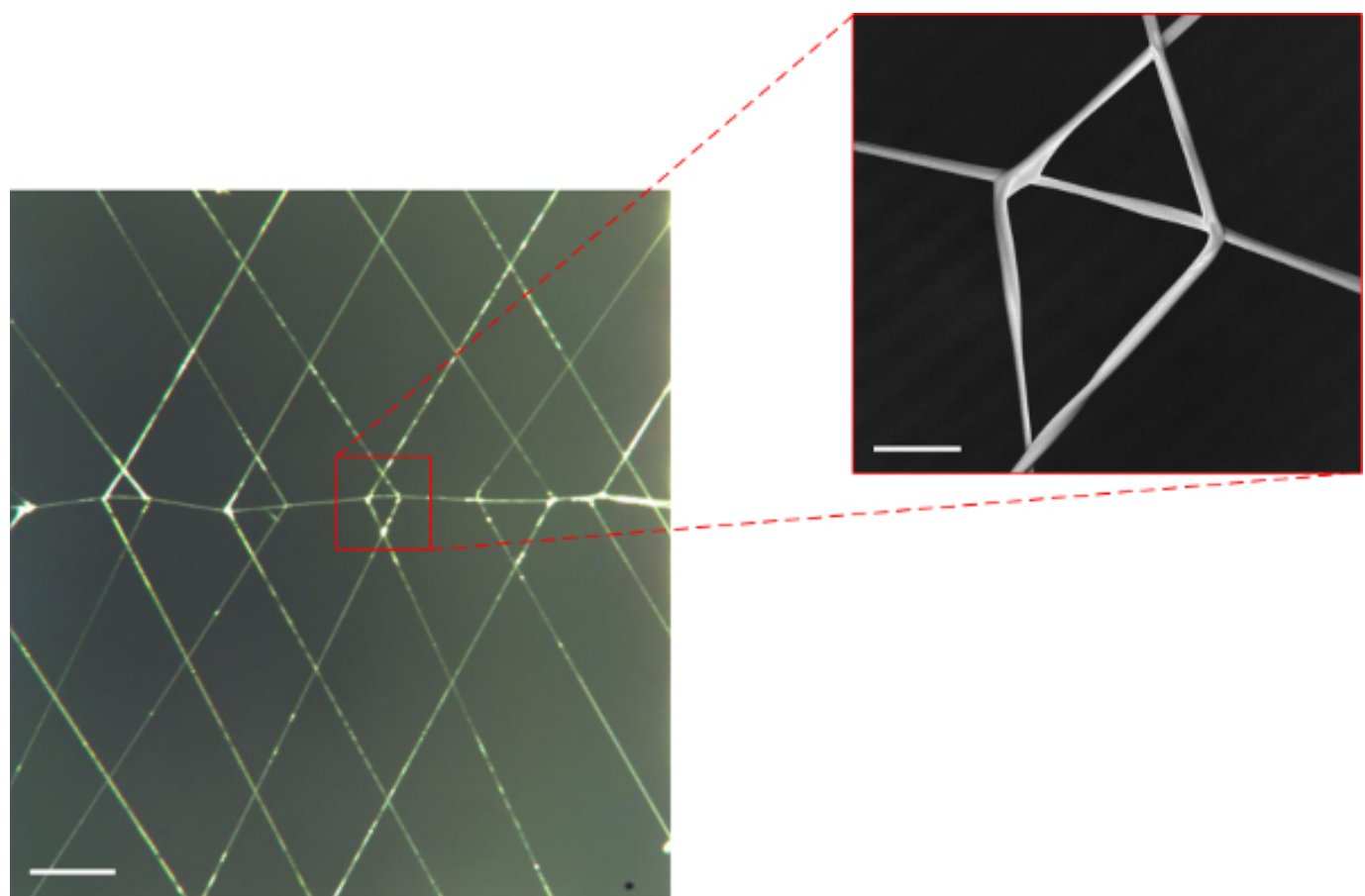

Figure 8. (A) Optical image of fabricated "dual chevron" suspended web structure having 1 support fiber (horizontal) and 22 bifurcated branched PMMA fibers (15X optical magnification; scale bar $=1 \mathrm{~mm}$ ), (B) SEM image of a single bifurcated fiber $(80 \mathrm{X}$ magnification; scale bar $=100 \mu \mathrm{m})$ Please click here to view a larger version of this figure.

Figure 9 displays close up view of the sequential point-to-point order of fiber fabrication at which the 3-axis robot and dispensing system can generate the freely suspended web-like structures. The polymer contact points displayed with numbers correspond to the programmed initiating and terminating points in the JR-C software referenced in Figure 3B above. The arrows represent robot trajectory. Figure 10 displays micron and submicron polymer fibers, which demonstrates the direct write system's ability to fabricate wires of varying sizes. 


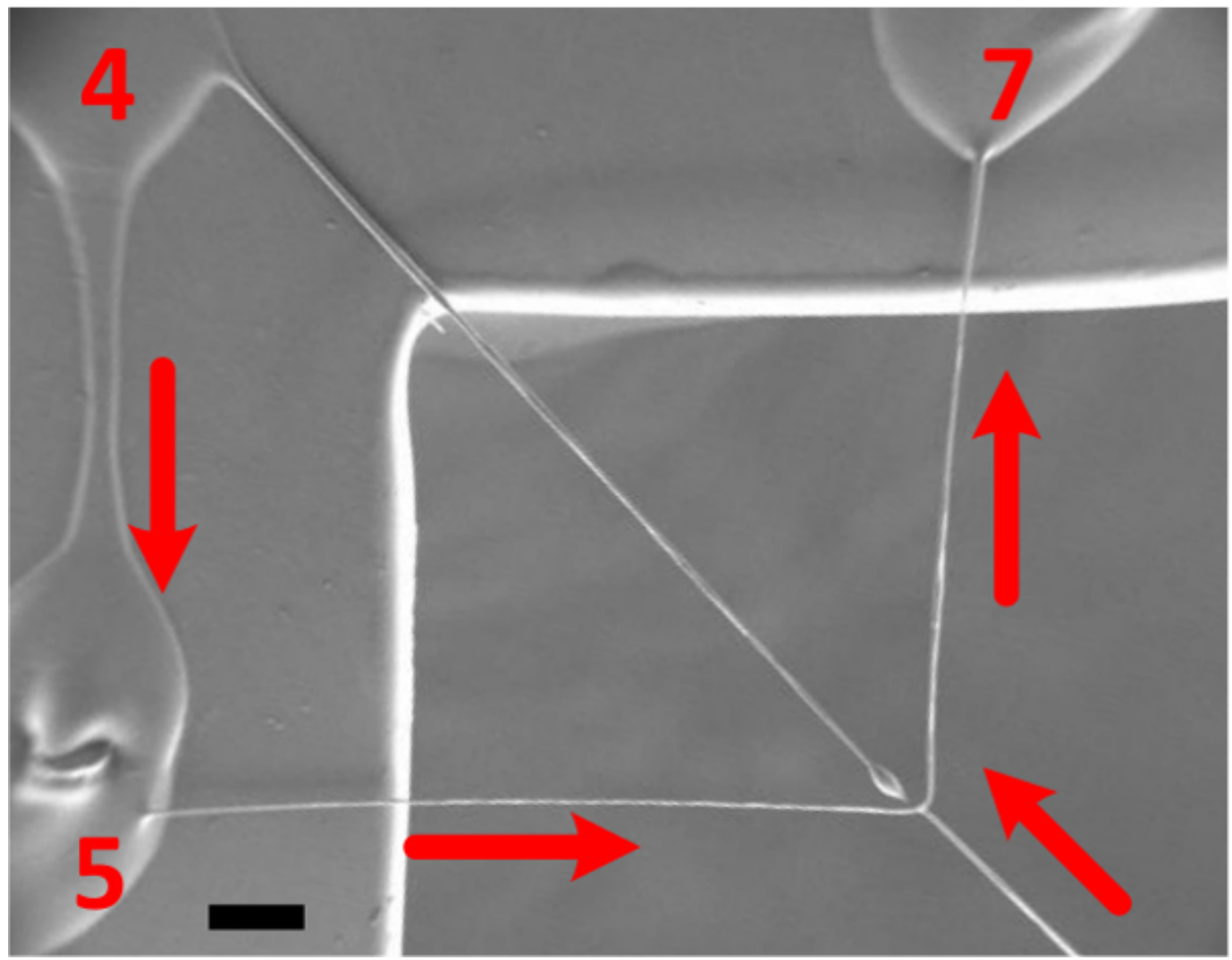

Figure 9. SEM image illustrating the sequential point-to-point fabrication order for drawing the micron and submicron polymer fibers $(\mathbf{2 9 X}$ magnification; scale bar $=\mathbf{2 0 0} \boldsymbol{\mu m})$. Please click here to view a larger version of this figure.

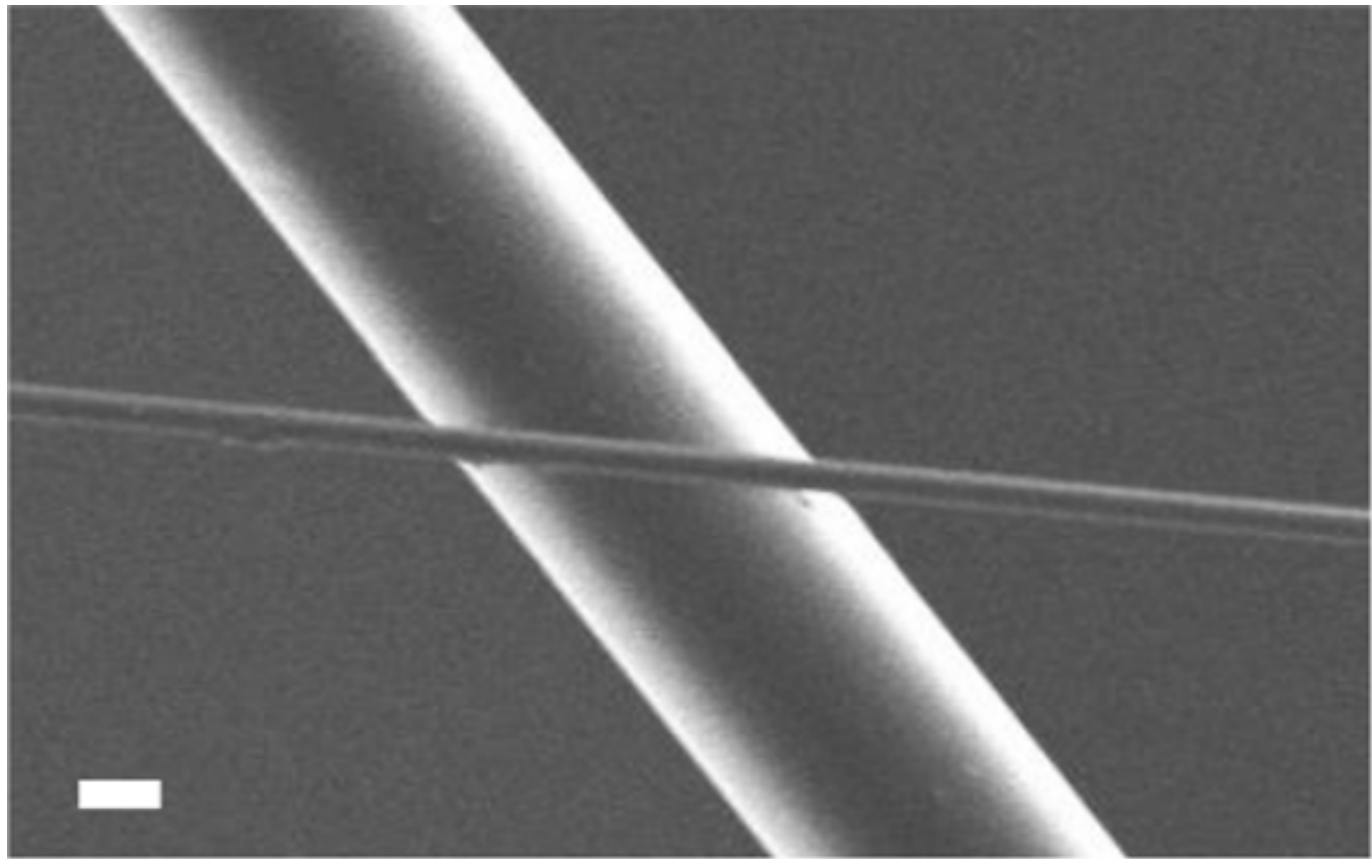

Figure 10. SEM image of micron $(6.5 \mu \mathrm{m})$ and sub-micron $(555 \mathrm{~nm})$ fibers drawn using a $20 \%$ concentration PMMA polymer solution $(2,270 X$ magnification; scale bar $=\mathbf{2} \boldsymbol{\mu m})$. Please click here to view a larger version of this figure.

Polymer fiber length and diameter were measured using scanning electron microscopy. Table 1 shows the average wire diameter for each unique structure presented in Figures 6-8 above corresponding to the bifurcated branch fibers and the support fibers. Table 2 displays the polymer parameters that were measured from the $24 \%$ PMMA solutions that were utilized to fabricate the structures noted above.

\begin{tabular}{|l|l|l|l|}
\hline & Structure diameter in Figure $\mathbf{6}$ & Structure diameter in Figure 7 & Structure diameter in Figure $\mathbf{8}$ \\
\hline
\end{tabular}




\begin{tabular}{|l|l|l|l|}
\hline Support fibers & $8.65 \pm 1.43 \mu \mathrm{m}$ & $9.39 \pm 1.23 \mu \mathrm{m}$ & $9.31 \pm 1.65 \mu \mathrm{m}$ \\
\hline Branch fibers & $20.96 \pm 3.35 \mu \mathrm{m}$ & $15.92 \pm 1.44 \mu \mathrm{m}$ & $12.24 \pm 5.42 \mu \mathrm{m}$ \\
\hline
\end{tabular}

Table 1. Average diameters of the suspended support and branch fibers shown in Figures 6-8 for a $24 \%$ PMMA solution.

\begin{tabular}{|l|l|}
\hline & $24 \%$ PMMA \\
\hline Viscosity (Pa*sec) & 35.19 \\
\hline Surface Tension (mN/m) & 262.01 \\
\hline Mass Transfer Coefficient (m/sec) & $8.59 \times 10^{-8}$ \\
\hline
\end{tabular}

Table 2. Polymer parameters of the $24 \%$ PMMA solutions that were utilized to fabricate the fiber structures presented in this work.

\section{Discussion}

Before attempting each trial, it is critical that the viscosity, mass transfer coefficients and surface tension test of the polymer solutions be accurately measured in order to determine whether the robot and dispensing system is capable of processing the desired polymer. As described previously by our group, the polymer solutions must retain adequate: 1) surface tension to enable the formation of liquid filaments into micron/ sub-micron structures; 2 ) viscosity to withstand capillary break up; and, 3) evaporation rate to enhance fiber solidification ${ }^{18}$. The synergy between these parameters is key to successfully produce fibers over a specified range of diameters. At the same time, instability in any of these parameters prevents the formation of micron/sub-micron scale fibers. To maintain the synergy between these parameters during fiber fabrication, it is important to ensure that the needle and needle valve are thoroughly cleaned after a direct-write session to prevent: 1) contamination of the solution; 2) a reduction in the polymer solution flow rate through the needle; and, 3) excessive growth in the polymer bead at the tip of the needle. In addition, the temperature-controller on the heater must be set to the desired temperature to maintain a constant evaporation rate of the polymer solution.

The bifurcated branch fibers were $59 \%, 41 \%$ and $24 \%$ larger in diameter than that of the support structures in Figures 6-8, respectively, using the $24 \%$ PMMA solution. This is primarily due to the distance at which the fibers are drawn. Specifically, the support structures are drawn across the entire width of the substrate $(10.0 \mathrm{~mm}$ in $X$ and $Y$ directions; $14.4 \mathrm{~mm}$ diagonally). As a result, these are the longest fibers of the overall suspended structures. The bifurcated branch structures are significantly shorter, ranging from $7 \mathrm{~mm}$ maximum length down to $2.5 \mathrm{~mm}$. This shorter fiber drawing length does not effectively induce the fiber strain required during the fiber thinning process in order to produce the small diameter fibers. On the other hand, larger diameter wires are required to serve as the support fibers in order to effectively sustain the tugging and deformation induced during the bifurcated branch drawing process. As the bifurcation branches are drawn across the support fibers, a remodeling of the support fiber geometry can occur due to the drawing forces as well as a localized dissolution of the PMMA polymer at the interface between the support and branched fibers from the solvent present in the polymer solution. Thus, in some instances, the support fibers may need to be fabricated from a polymer solution consisting of a higher concentration of polymer to produce larger diameter and mechanically stronger fibers.

There are primarily three effective methods for modifying the existing protocol to generate a wider range of support and branched fiber diameters: 1) initially dispense the polymer from a larger needle tip (e.g., $25 \mathrm{G}$; ID = $254 \mu \mathrm{m}$ ) to generate the support fibers and then exchange for a smaller needle tip (e.g., $32 \mathrm{G} ; \mathrm{ID}=101.6 \mu \mathrm{m})$ to fabricate the smaller branched fibers; 2 ) as noted above, use multiple polymer concentrations; and/or, 3) adjust the feed rate, i.e., the speed at which the stage traverses, where increasing the feed rate produces smaller diameter fibers and decreasing the feed rate creates larger diameter fibers. To date, we have been able to successfully fabricate fibers as small as $90 \mathrm{~nm}$; however, the fiber yield at this dimension is low due to capillary breakup.

One limitation of the automated direct write process is that only one concentration of polymer solution can be dispensed at a time. This restricts the level of complexity of the suspended structures to be developed without having to: 1) add a second dispensing valve to the robot; or, 2) remove the existing valve and perform the cleaning protocol (Section 3.4) before dispensing the second polymer solution, which takes additional time. A second limitation is the feed rate (or print speed), where the maximum feed rate that the system is capable of achieving is $500 \mathrm{~mm} / \mathrm{sec}$. However, there is a tradeoff between feed rate and fiber formation. Specifically, if the inertial forces (forces due to the feed rate) are greater than the surface tension forces and evaporation rate of the polymer solution, fiber formation does not occur. On the other hand, if the feed rate is too low, fibers will fracture due to excessive evaporation before and during the elongation process. Thirdly, fiber and structure dimensions are limited to the operating range of the stage of the robot, i.e., $200 \mathrm{~mm}, 200 \mathrm{~mm}$ and $25 \mathrm{~mm}$ (with a $10 \mu \mathrm{m}$ positional accuracy) in the $x, y$ and $z$ directions, respectively. Nonetheless, this process does enable the formation of high aspect ratio (fiber length:diameter) fibers. Strategically varying the dispensing tip size and the polymer solution concentration would allow for a broader fiber diameter range, creating the ability to generate freely suspended structures of higher complexity.

By following the protocols stated above, micron and submicron diameter polymer fibers can be generated with a high level of spatial control by exploiting the surface tension-driven fluid mechanics of the polymer solutions, which cannot be controlled with any other wet, dry or electrospinning processes. From prior work ${ }^{8,19}$, we know that this technique can be utilized to fabricate intricate micro/submicron fluidic devices ${ }^{19}$ and bioengineered scaffolds ${ }^{8}$. This cheap and easy technique has its own advantages over traditional planer fabrication methods in many ways.

\section{Disclosures}

The authors have nothing to disclose. 


\section{Acknowledgements}

This work is supported by the NSF-EPSCoR (grant \#0814194) and the Department of Bioengineering.

\section{References}

1. Andrew, K. E., Glenn, D. P., Simon, M. C., Dietmar, W. H. Combining Electrospun Scaffolds with Electrosprayed Hydrogels Leads to ThreeDimensional Cellularization of Hybrid Constructs. Biomacromolecules. 9, (8), 2097-2103 (2008).

2. Cho, S. M., Kim, Y. J., Kim, Y. S., Yang, Y., Ha, S. -C. The application of carbon nanotube-polymer composite as gas sensing materials. Sensors. Proc. of IEEE. 2, 701-704 (2004).

3. Xu, F., Horak, P., Brambilla, G. Optical microfiber coil resonator refractometric sensor. Optics Express. 15, (12), 7888-7893 (2007).

4. Dalton, P. D., Joergensen, N. T., Groll, J., Moeller, M. Patterned melt electrospun substrates for tissue engineering. Biomed. Mater. 3, (3), 034109 (2008)

5. Hadjizadeh, A., Doillon, C. J., Vermette, P. Bioactive polymer fibers to direct endothelial cell growth in a three-dimensional environment. Biomacromolecules. 8, (3), 864-873 (2007).

6. Xu, C. Y., Inai, R., Kotaki, M., Ramakrishna, S. Aligned biodegradable nanofibrous structure: a potential scaffold for blood vessel engineering. Biomaterials. 25, (5), 877-886 (2004).

7. You, Y. M., Lee, B. L. eeS. J., Lee, T. S., Park, H. In vitro degradation behavior of electrospun polyglycolide, polylactide, and poly(lactide-coglycolide). J. Appl. Polym. Sci. 95, (2), 193-200 (2005).

8. Berry, S., et al. Endothelial cell scaffolds generated by 3D direct writing of biodegradable polymer microfibers. Biomaterials. 32, (7), 1872-1879 (2011).

9. Dalton, A. B., et al. Super-tough carbon-nanotube fibres. Nature. 423, 703 (2003).

10. Dalton, A. B., et al. Continuous carbon nanotube composite fibers: properties, potential applications, and problems. J. Mater. Chem. 1-3 (2004).

11. Sperling, L. H. Introduction to physical polymer science. 325-347 Wiley (1992).

12. Harfenist, S. A., et al. Direct drawing of suspended filamentary micro- and nanostructures from liquid polymers. Nano. Lett. 4, (10), 1931-1937 (2004).

13. Yang, R. R., He, J. H., Xu, L., Yu, J. Y. Bubble-electrospinning for fabricating nanofibers. Polymer. 50, (24), $5846-5850$ (2009).

14. Berry, S., et al. Characterization and modeling of direct-write fabrication of microscale polymer fibers. Polymer. 52, (7), 1654-1661 (2011).

15. Janome Desktop Robot JR2000N Series Operation Manual. Janome Sewing Machine Co Tokyo, Japan (2007).

16. Brookfield DV-II+ Programmable Viscometer Operating Manual, Manual No. M/97-164-B299. Middleboro, MA (1999).

17. Flegler, S. L., Heckman, J. W., Klomparens, K. L. Scanning and transmission electron microscopy : an introduction. W.H. Freeman (1993).

18. Berry, S. M., Pabba, S., Cohn, R. W., Keynton, R. S. Direct-Write Drawing of Carbon Nanotube/Polymer Composite Microfibers. J. Nanomater. 2012, 1-8 (2012).

19. Berry, S. M., Roussel, T. J., Cambron, S. D., Cohn, R. W., Keynton, R. S. Fabrication of suspended electrokinetic microchannels from directlywritten sacrificial polymer fibers. Microfluid. Nanofluid. 13, 451-459 (2012). 